\title{
Elicitation of Antimicrobial Active Compounds by Streptomyces-Fungus Co-Cultures
}

\author{
Matthieu Nicault ${ }^{1,2}$, Ali Zaiter $^{3}{ }^{\circ}$, Stéphane Dumarcay ${ }^{4}$, Patrick Chaimbault ${ }^{3}{ }^{\circledR}$, Eric Gelhaye ${ }^{2}$, \\ Pierre Leblond ${ }^{1, *}$ (1) and Cyril Bontemps ${ }^{1, *}$ \\ 1 Université de Lorraine, INRAE, DynAMic, F-54000 Nancy, France; matthieu.nicault@univ-lorraine.fr \\ 2 Université de Lorraine, INRAE, IAM, F-54000 Nancy, France; eric.gelhaye@univ-lorraine.fr \\ 3 Université de Lorraine, LCP-A2MC, F-57000 Metz, France; ali.zaiter@univ-lorraine.fr (A.Z.); \\ patrick.chaimbault@univ-lorraine.fr (P.C.) \\ 4 Université de Lorraine, INRAE, LERMAB, F-54000 Nancy, France; stephane.dumarcay@univ-lorraine.fr \\ * Correspondence: pierre.leblond@univ-lorraine.fr (P.L.); cyril.bontemps@univ-lorraine.fr (C.B.)
}

Citation: Nicault, M.; Zaiter, A.; Dumarcay, S.; Chaimbault, P.; Gelhaye, E.; Leblond, P.; Bontemps, C. Elicitation of Antimicrobial Active Compounds by Streptomyces-Fungus Co-Cultures. Microorganisms 2021, 9 , 178. https://doi.org/10.3390/ microorganisms 9010178

Received: 10 December 2020

Accepted: 12 January 2021

Published: 15 January 2021

Publisher's Note: MDPI stays neutral with regard to jurisdictional claims in published maps and institutional affiliations.

Copyright: (c) 2021 by the authors. Licensee MDPI, Basel, Switzerland. This article is an open access article distributed under the terms and conditions of the Creative Commons Attribution (CC BY) license (https:/ / creativecommons.org/licenses/by/ $4.0 /)$.

\begin{abstract}
The bacteria of the genus Streptomyces and Basidiomycete fungi harbor many biosynthetic gene clusters (BGCs) that are at the origin of many bioactive molecules with medical or industrial interests. Nevertheless, most BGCs do not express in standard lab growth conditions, preventing the full metabolic potential of these organisms from being exploited. Because it generates biotic cues encountered during natural growth conditions, co-culture is a means to elicit such cryptic compounds. In this study, we explored 72 different Streptomyces-fungus interaction zones (SFIZs) generated during the co-culture of eight Streptomyces and nine fungi. Two SFIZs were selected because they showed an elicitation of anti-bacterial activity compared to mono-cultures. The study of these SFIZs showed that co-culture had a strong impact on the metabolic expression of each partner and enabled the expression of specific compounds. These results show that mimicking the biotic interactions present in this ecological niche is a promising avenue of research to explore the metabolic capacities of Streptomyces and fungi.
\end{abstract}

Keywords: Streptomyces; fungus; co-culture; specialized metabolism; biosynthetic gene cluster; antimicrobial agents

\section{Introduction}

Microorganisms form complex multispecies communities in all environments (e.g., soil, oceans, microbiota) where they play key roles. In soil, they contribute to homeostasis by participating in biogeochemical cycles [1] or by improving nutrient availability for plants [2]. Bacteria and fungi of a same community are in constant interaction (commensalism, mutualism, competition or antagonism) that can affect their growth or induce more specific behaviors, such as pathogenicity [3]. These bacterial-fungal interactions (BFIs) are often driven by the production of specialized metabolites (SMs). The latter can have a direct impact (e.g., antibiosis, sugar degradation) but can also act as communication signals and trigger expression of other specific biosynthetic pathways in other niche inhabitants [4]. Among these SMs, some exhibit interesting biological properties (e.g., antimicrobials) and represent a source for the conception of new drugs [5].

In that respect, fungi and bacteria of the genus Streptomyces are of special interest, as they provide alone $42 \%$ and $32 \%$ of the natural products used in the human and veterinary medicine, respectively [6]. For Streptomyces, genomic analyses have shown that they possess between 20 and 50 biosynthesis gene clusters (BGCs) predicted to produce SMs [7,8]. Yet, Streptomyces strains are generally used for the production of a single or a few compounds. For example, Streptomyces griseus, the producer of the antibiotic streptomycin [9] and Streptomyces avermitilis, the producer of the polyketide anthelmintic avermectin [10] possess 34 and 25 BGCs, respectively [11,12]. The genetic model Streptomyces coelicolor A3(2), for 
its part, was known for a long time to encode only three antibiotics (the aromatic polyketide antibiotic actinorhodin, oligopyrrole prodiginine antibiotics, and the non-ribosomal peptide calcium-dependent antibiotic) [13,14], although its genome contains 20 BGCs [13]. The access to the genome sequences of many other species since has confirmed that the potential to code for specialized metabolites far exceeds the activities and compounds that can be identified based on bioassays or the detection of direct molecules, and that most BGCs remain silent in standard laboratory conditions [15].

It is therefore important to find new methods to explore and to induce the production of specialized metabolites by activating these biosynthetic pathways [16,17], and many methods were developed for that purpose over the past decades. For instance, it has been successfully achieved by rewiring regulatory networks [18], ribosome engineering, regulatory unlocking, heterologous expression [19-21], or by randomly modifying parameters of culture conditions (e.g., nutrient source, culture medium) [22]. In recent years, there has been a renewal of interest to express cryptic SMs in the lab through co-culture approaches [23]. Biotic stresses are indeed likely to trigger adaptive responses (e.g., competitive weapons, hormones, communication molecules) by mimicking signals emitted by community co-inhabitants [24].

Co-cultures were described with various bacteria and fungi [23]. Regarding fungi, wood-decaying fungi have already been used $[25,26]$ and are of special interest as they are known to control and to modify bacterial communities in their environment [27,28], in particular through metabolites production and $\mathrm{pH}$ modification [29]. They thus constitute good candidates to produce antibacterial compounds or to trigger such production in interaction.

For bacteria, as Streptomyces remain one of the main source of SMs, they were often co-cultivated together $[30,31]$ or with other bacterial genera [32-35]. Streptomyces-fungi cocultures are on the other hand rarer, despite having showed promising results. For instance, co-cultures of Aspergillus fumigatus MR2012 with Streptomyces leeuwenhoekii C34 [36] or Streptomyces rapamycinicus and the model fungus Aspergillus nidulans [37] led to the production of new compounds.

The aim of our work was to exploit a Streptomyces strain collection isolated from the same soil microhabitat in co-cultures with different wood-decaying fungi in order to stimulate the production of antibacterial compounds. Bi-partite co-cultures were set up and the antimicrobial activity was assessed against indicator bacterial strains (mostly Bacilli). Monitoring the antimicrobial activity was used as a screening method throughout the analytical processes. Here we observed an important induced chemical diversity that explains some mechanisms of microbial interactions. Activity-guided separation led to the detection of candidate compounds that could be responsible for the induced antimicrobial activities.

\section{Results and Discussion}

\subsection{Induction of Antibacterial Activities by Streptomyces-Fungus Co-Culture}

To set up bacterial-fungal co-cultures, we used two collections of the soil dwelling bacterium Streptomyces and of forest basidiomycetes available in our laboratories (Figure 1). On the one hand, the bacterial collection was made up of eight Streptomyces isolated from the same forest soil micro-habitats and belonging to identical or different species as previously described by Nicault et al. [8]. Their genome sequences were determined and showed a great diversity of their specialized metabolite BGCs, with 261 identified for the eight strains [8]. On the other hand, the fungal collection was made up of wooddecaying basidiomycetes. Besides the well-known ability of wood-decaying fungi to control bacterial communities [38,39], the fungi have been chosen for their potential to produce antimicrobial products [40-44], for instance the genera Trametes [45] or Stereum [46]. In our experimental design, bacterial and fungal couples were grown on agar plates at each side of the petri dishes (Figure 1A) generating a Streptomyces-fungus interaction zone (SFIZ). A similar design was previously used to reveal compounds induced during co-cultures, 
for instance with marine fungi [47]. Our case had the advantage to enable the interaction either by a diffusible signal in the agar or through contact between the two partnerstwo interaction types already described in Streptomyces and fungal co-cultures $[48,49]$. As nutritive sources can have a profound effect on specialized metabolism expression, we only used the GA medium that is commonly used for Streptomyces culture. Our initial collection consisted of eight Streptomyces and nine fungi and all of them with the exception of Phaeolus schweinitzii were able to grow on GA medium, resulting in 64 different cocultures (Figure 1B). In order to survey the impact of the co-culture on the metabolome, we focused on metabolites that were expressed specifically through co-culture, that is to say those that remained cryptic in mono-cultures. For that purpose, we tested the inhibition activity of the ethyl acetate extract of the inhibition zone in comparison to the mono-culture controls against the indicator Bacillus subtilis ATCC6633 strain (Figure 1B). Each interaction was screened once and only experiments showing an elicitation by co-culture of the antiBacillus activity were repeated five times. Two out of 64 interactions revealed co-culture elicitations (Figure 1B). This number is in the same range as reported by Schroeck et al. [37], where elicitation was found in one interaction out of 58 between Aspergillus nidulans and different Streptomyces. In our case, most controls, either bacterial or fungal, showed activity against $B$. subtilis ATCC6633 and prevented the revealing of a potential elicitation during the screening. This result confirms that fungi and Streptomyces are prominent sources of bioactive molecules and we can speculate that the number of elicitations is probably underestimated here.

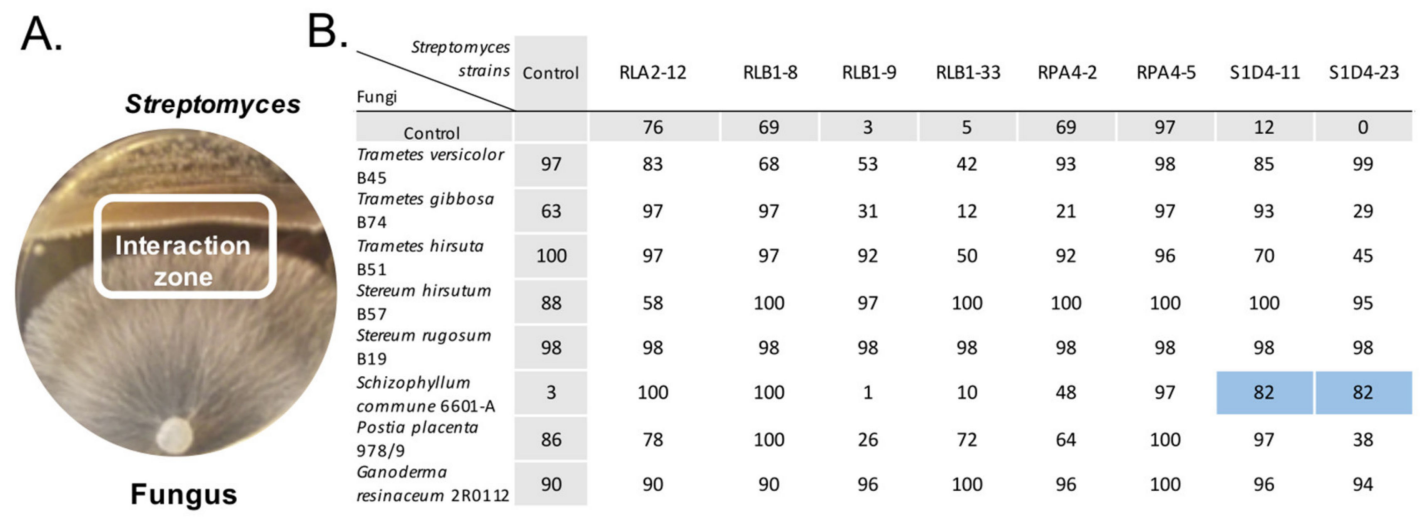

Figure 1. Streptomyces-fungus co-culture setup and screening. (A) Illustration of a Streptomyces-fungus co-culture after 14 days of growth. (B) Screening of bioactive molecules elicited during the co-culture. The compound extracts of monocultures of fungi and Streptomyces (rows and columns named "control") in GA medium were compared with the extracts resulting from their co-culture in a bioassay experiment against the growth of B. subtilis ATCC6633. The values in the table indicate the percentage of inhibition of B. subtilis ATCC6633 after a $24 \mathrm{~h}$ growth period in comparison with a control grown in absence of the extract. The two co-cultures between S. commune 6601-A with S1D4-11 and S1D4-23 (highlighted in blue) were selected as they presented a significant $(t$-test $p$ value $<0.05)$ impact on the growth of $B$. subtilis ATCC6633 in comparison with the controls.

The two selected couples were both composed of the fungus S. commune 66-01A with Streptomyces S1D4-11 or S1D4-23. S. commune is a wood-decaying fungus that can induce melanin, indole, flavonoids, and carotenoids when grown in the presence of co-occurring bacteria or fungi [50]. It also represents a good interaction model with Streptomyces, as it was shown to restore hyphal formation-deficient phenotypes in some strains [51]. S1D411 and S1D4-23 are closely related but different species of Streptomyces and each were previously predicted to harbor 36 BGCs with 25 in common and 11 specific ones [8]. It can be noticed that the two other Streptomyces RLB1-33 and RLB1-9 that showed no anti-B. subtilis activity in mono-culture (Figure 1B) had no induced capacity in their SFI zones with S. commune. This result highlights that whether the elicited activity came from the fungus or the bacteria, it was specific between S. commune and the strains S1D4-11 and S1D4-23. 
The interactions with elicitation against B. subtilis ATCC6633 were named S1D4-11-FIZ and S1D4-23-FIZ. They were tested against three additional environmental Bacillus strains, isolated from the same soil as the Streptomyces collection (Figure 2), and strains RB2-2N12 and RB2-2N10 appeared sensitive to the SFIZ extracts though at different levels in comparison with the controls. Antibacterial activity of S1D4-11-FIZ and S1D4-23-FIZ extracts were also tested against other indicator strains: Staphylococcus aureus ATCC29213, Escherichia coli ATCC25922, Enterococcus faecalis ATCC292212, Pseudomonas aeruginosa ATCC27853, Acinetobacter baumanii ATCC19606, Klebsiella pneumonia ABC42, and Enterobacter cloacae ABC291. No significant activity was detected against these bacterial species Although we did not perform an extensive screening and only revealed anti-Bacillus activities, our results show that co-culture can revealed antibacterial activities not expressed in standard lab conditions. This extended approach, when applied to other bacteria of interest, such as antibiotic multi-resistant bacteria, should provide a simple and generalizable means of discovering new biomolecules.

A.

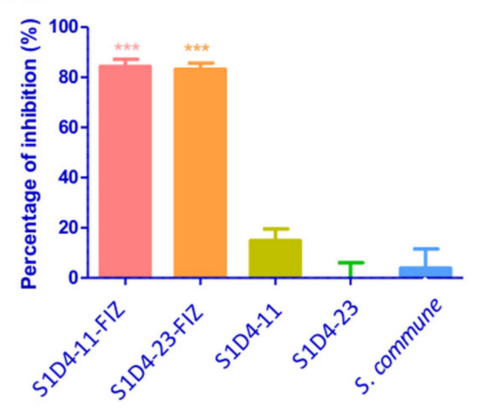

C. Bacillus sp. RB2-2N10

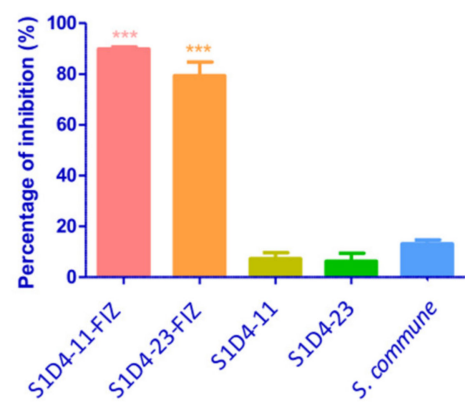

B. Bacillus sp. RB2-2N12

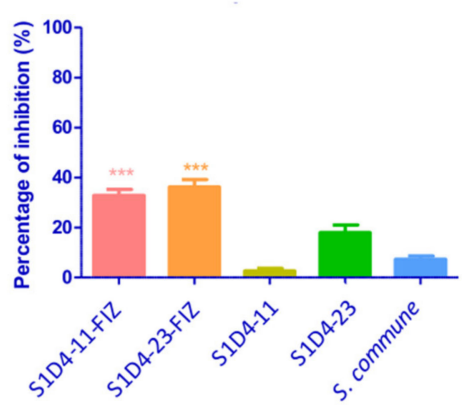

D. Bacillus sp. RB2-1N16

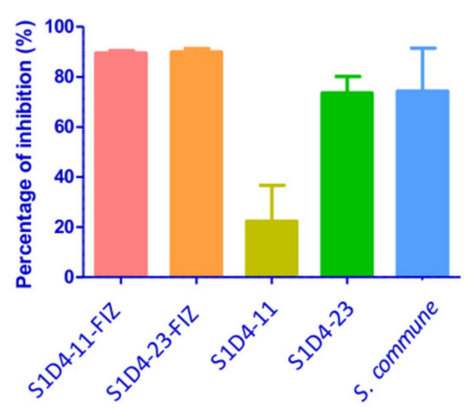

Figure 2. Antimicrobial activities of S1D4-11-FIZ and S1D4-23-FIZ against different Bacilli. Inhibition was quantified as a percentage of growth inhibition in comparison with a control without extract. The growth was measured by spectrometry at OD $600 \mathrm{~nm}$ after $24 \mathrm{~h}$ of growth. The different Bacilli strains are (A) Bacillus subtilis ATCC6633, (B) Bacillus sp. RB2-2 N12, (C) Bacillus sp. RB2-2 N10, and (D) Bacillus sp. RB2-1 N16. Statistical difference was assessed with a $t$-test. ${ }^{* * *}=p$ value $<$ 0.005 for both comparisons between fungus and Streptomyces-fungus interaction zone (SFIZ) and Streptomyces and SFIZ.

\subsection{Impact of Co-Culture on Metabolome}

In order to decipher the metabolic impact of co-culture, molecular profilings of the two selected SFIZs as well as the corresponding pure culture extracts were analyzed by gas chromatography coupled to a mass spectrometer (GC-MS). GC-MS was already used for the characterization of bioactive Streptomyces strains [52] and the used derivatization enabled us to detect more compounds, such as amino acids and lipids. Based on ions extracted from mass spectra, 2796 features were detected with MzMine 2 software and partial least square-discriminant analysis (PLS-DA) showed that each extract (controls and SFIZs) can be discriminated from the others (Figure 3A). From the PLS-DA, discriminating 
variables (26\%) were compared in a heatmap (Figure 3B) and only showed slight overlap, giving a unique bar code for each condition. These results show that mono-culture extracts are different and that each microbe has a specific metabolomic signature. Secondly, the extracts from the co-cultures differed from their controls, indicating that a specific production of compounds was induced during the interaction. To confirm these results with more polar and soluble compounds [53], SFIZ and control extracts were analyzed by ultra-high-performance liquid chromatography with tandem mass spectrometry (LCMS/MS). In the co-culture experiment between S1D4-11 and S. schizophyllum, 387 features were specific to the fungus extract (i.e., not found in other extracts) and 163 to the bacterial extract (Figure 4). In the co-culture between S1D4-23 and S. schizophyllum, 340 features were specific to the fungus extract and 131 to the bacterial extract. Regarding the SFIZs of these two co-cultures, 458 and 473 features were respectively identified, with around a quarter being shared with either the fungal or the bacterial controls, logically reflecting that they are produced during both mono- and co-cultures. Interestingly, a greater proportion of these SFIZ features were specific (225 for S1D4-11-FIZ and 202 for S1D4-23-FIZ) and confirmed that co-cultures greatly affect the metabolome. Moreover, almost half of these SFIZ specific features $(n=108)$ were shared between S1D4-11-FIZ and S1D4-23-FIZ, suggesting that a significant part of the specific metabolic response induced by the interaction is common between the two co-cultures. We can speculate that most of these shared compounds are produced by S. commune, which is common to both co-cultures, and/or by a common metabolism induced in Streptomyces in response to the fungus.

A.

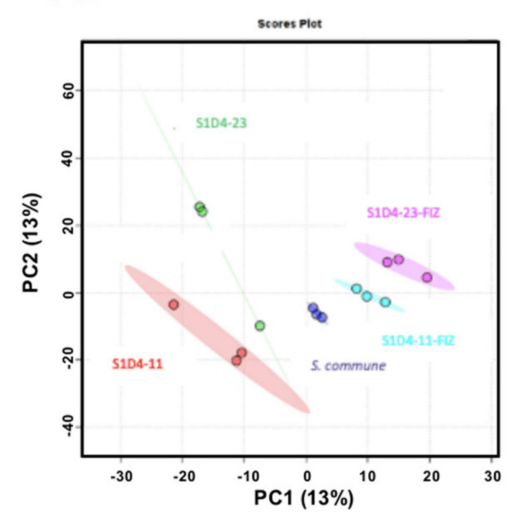

B.

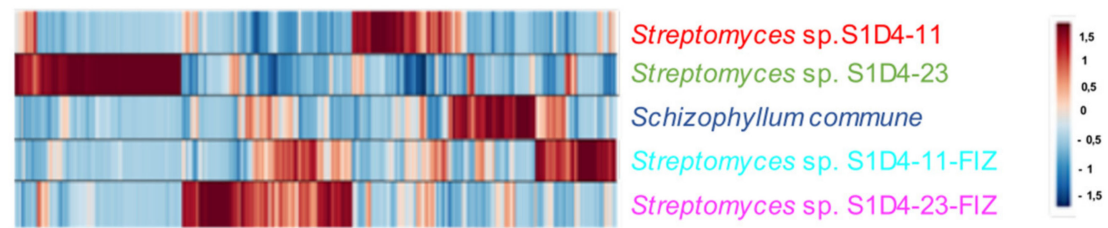

Figure 3. Gas chromatography-mass spectrometry (GC-MS) spectrum comparison. (A) Partial least square-discriminant analysis (PLS-DA)comparison of GC-MS spectra of mono- and co-cultures. (B) Heat-map of the first 750 discriminant features $(26 \%)$ revealed by PLS-DA. The scale indicates the relative abundance of features calculated by centered-reduced of initial intensity.

\subsection{Towards the Identification of Potential Bioactive Compounds}

To identify the chemical actors of these specific metabolic responses, a LC-MS molecular networking with GNPS (Global Natural Products Social Molecular Networking) that gathers MS/MS spectra into networks corresponding to chemical families was performed. In total, 187 networks were detected with 159 identified as chemical families (Figure 5). The non-identified molecule families (labeled "no matches") could correspond to new molecules, and half of SFIZ compounds (nodes within networks) were present in this category. It might suggest that the co-cultures induced a less characterized metabolism than single cultures that can be the source of innovative molecules. Four networks were found to be specific to one or both SFIZs. Among them, S1D4-11-FIZ presented specific oligosaccharides, S1D4-23-FIZ presented phytoceramides and vinyl bromides, and both SFIZs had specific alkaloids and derivative compounds that can have pharmaceutical properties, including antibacterial activities [54]. 


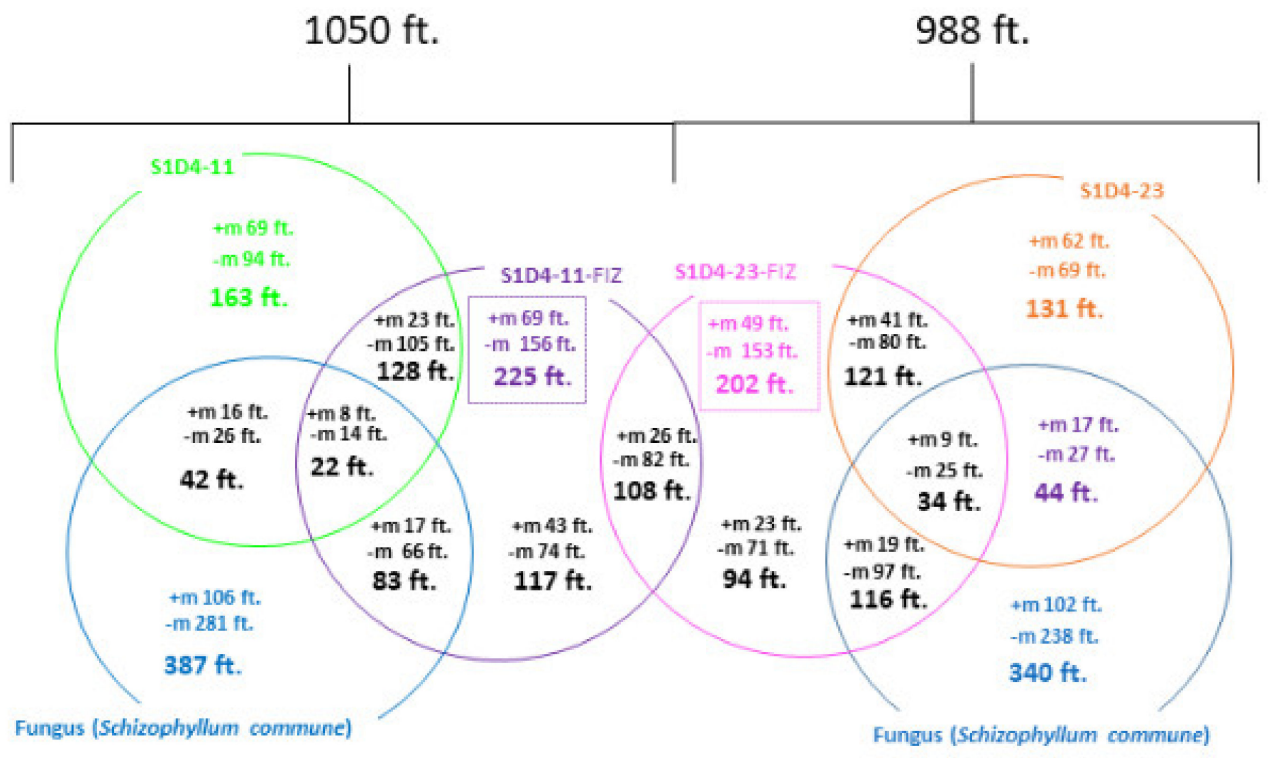

Figure 4. Distribution of common and specific features between SFIZs and controls. LC-MS metabolite profiles were recorded and analyzed with GNPS. The Venn diagram compares specific and common features between each SFIZ and its controls as well as SFIZ features between the two experiments. The number of SFIZ specific features for each experiment is indicated in the dashed square. $+\mathrm{m}$ : positive mode; $-\mathrm{m}$ : negative mode; ft.: features. 

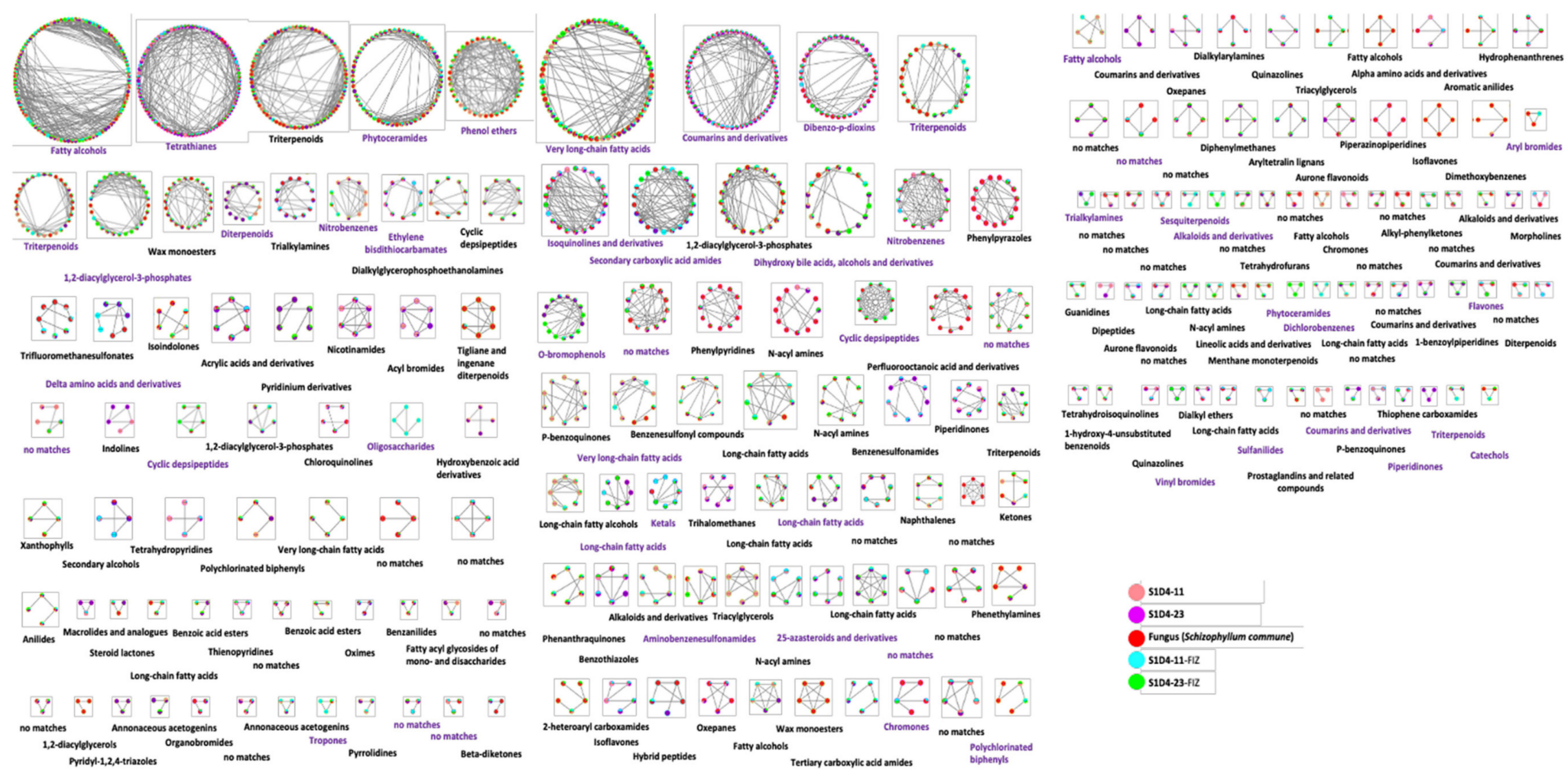

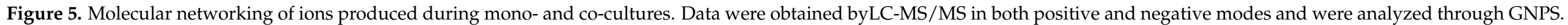

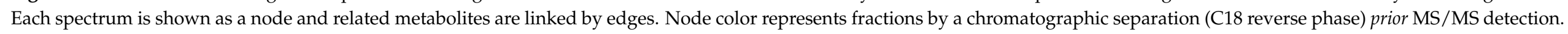

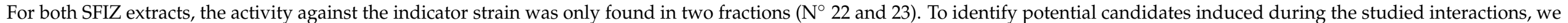

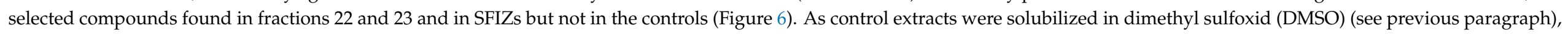

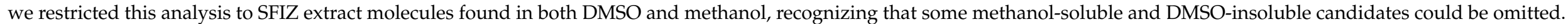




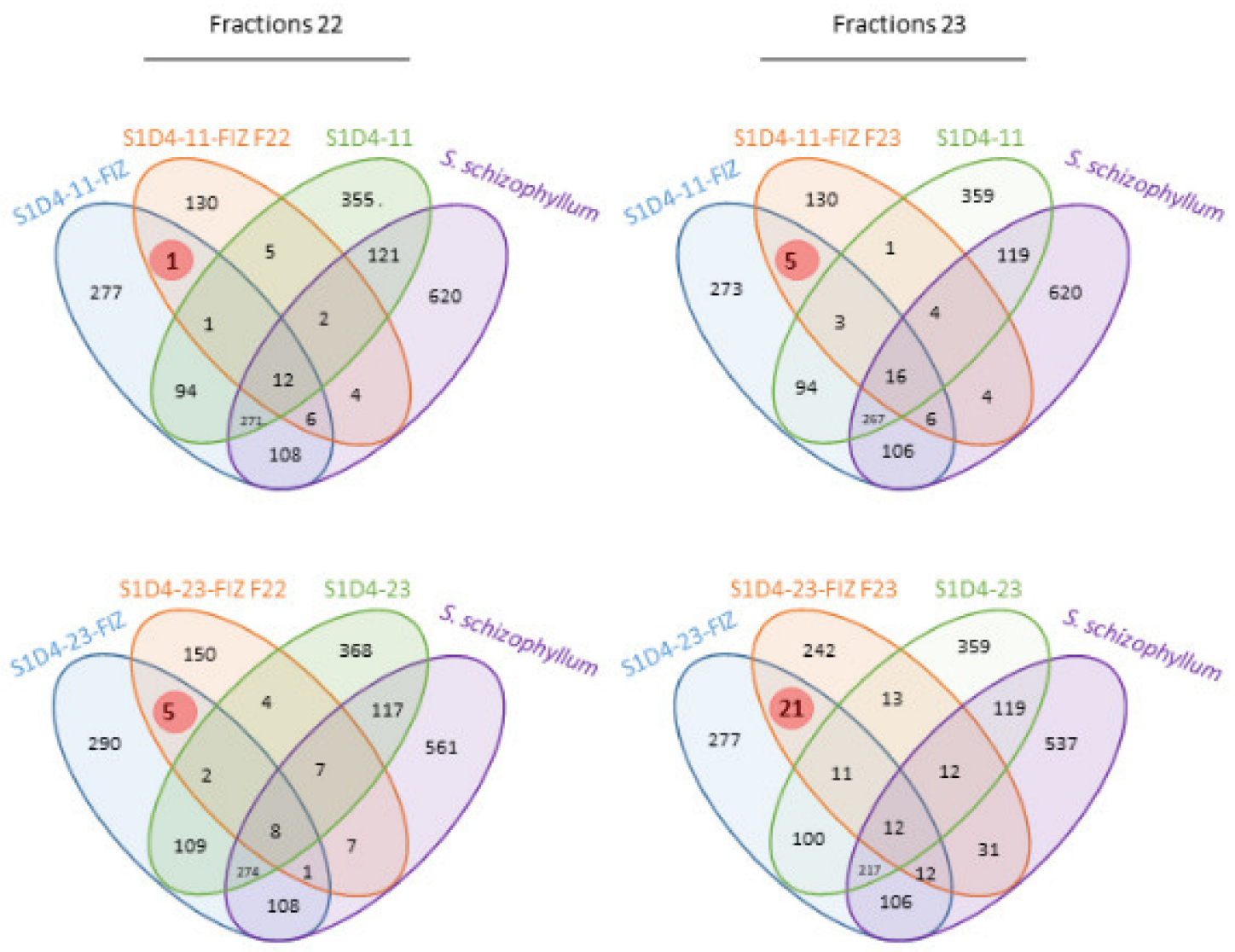

Figure 6. Identification of specific compounds in bioactive fractions. LC-MS spectra of fractions $\mathrm{N}^{\circ} 22$ and $\mathrm{N}^{\circ} 23$ (named S1D4-11-FIZ F22 and S1D4-23-FIZ F23, respectively) with anti-Bacillus activity were compared with single culture controls and with total extracts of SFIZ in DMSO, which also has similar activity. Compounds with potential activities (highlighted in red) are those at the intersection of the total SFIZ extract and in the fraction considered. The number of features found in each condition is indicated. Features were analyzed and compared with DEREPLICATOR+ in order to build the Venn diagram.

Although the mass spectrometer used in this work has a low resolution that did not allow identifying compounds of interest through the determination of molecular formulae, the LC-MS and LC-MS/MS profiles enabled us to compare samples obtained in the different conditions of culture in terms of the presence and absence of some specific metabolites. Hence, for the four fractions, one specific peak was identified in fraction 22 of S1D4-11-SFIZ, five in fraction 23 of S1D4-11-SFIZ, five in fraction 22 of S1D4-23-SFIZ and 21 in fraction 23 of S1D4-23-FIZ. For each couple of co-cultures, no MS peak was common between the two fractions, indicating that different molecules are responsible for the anti-Bacillus inhibition activity, or that their concentration is too low in a fraction compared to the others detected by MS. Determining which partner produces these different compounds was not possible, but it can be noticed that a few peaks were common between the two SFIZs. As the fungal partner is the same in both co-cultures, this suggests that most of the compounds were produced by variable gene pools in Streptomyces or that the fungal response is different and specific depending on its partner.

Characterizing the molecule(s) responsible for the activity is undoubtedly the next challenge. Obtaining higher precision on the measure of the $\mathrm{m} / \mathrm{z}$ values using as a minimum a Q-TOF (Time of Flight), but even better an Orbitrap or FTICR mass spectrometer will allow the determination of molecular formulae of intact molecules and their fragments facilitating their characterization using local MS databases and/or the literature. If no matching is obtained from these new data, this could also mean that these compounds 
belong to the dark metabolome, whose exploration is promising for the discovery of new antibiotics. In this case, genome analysis coupled with genetics approaches will be used to target and knock-out specific BGCs previously identified [8]. The loss of activity in a mutant will enable identifying the bioactive molecules and decipher the black metabolome of our Streptomyces-fungus interactions.

\section{Conclusions}

Taken together, these results suggest that co-culture had a significant effect on the metabolic expression of both organisms and that the metabolome of the interaction is not the simple addition of compounds produced by the fungi and those of the bacteria. In order to best explore this important diversity of metabolites, we have chosen an approach guided by anti-Bacillus inhibition activity induced by co-culture, and tried to link metabolome and phenotype. Interestingly, even if the precise identification of one or several metabolites could not be fully achieved, we were able to show that bioactive fractions had specific molecules, which are potentially antimicrobial candidates. Our results confirm that co-culture coupled with an activity-targeted approach represents an excellent means of activating cryptic metabolites in the laboratory and could lead to the discovery of molecules of interest.

\section{Materials and Methods}

\subsection{Strains and Culture Conditions}

The bacterial and fungal strains used in this work are listed in Figure 1. The Streptomyces strains were stored at $-20{ }^{\circ} \mathrm{C}$ as spore suspensions in glycerol $20 \%$ and were grown on GA medium ( $15 \mathrm{~g}$ starch, $5 \mathrm{~g} \mathrm{NaCl}, 5 \mathrm{~g} \mathrm{KCl}, 0.5 \mathrm{~g} \mathrm{~K}_{2} \mathrm{HPO}_{4}, 0.5 \mathrm{~g} \mathrm{KNO}_{3}, 0.5 \mathrm{~g} \mathrm{MgSO}$, $15 \mathrm{mg} \mathrm{FeSO}$, and $15 \mathrm{~g}$ Bacto agar in $1 \mathrm{~L}$ of water adjusted to $\mathrm{pH} 7.2$ ). Bacillus strains were grown in lysogeny broth (LB) $(10 \mathrm{~g}$ tryptone, $5 \mathrm{~g}$ yeast extract, $10 \mathrm{~g} \mathrm{NaCl}$ for $1 \mathrm{~L})$ at $30^{\circ} \mathrm{C}$. Fungi were maintained on MA medium (15 g malt extract and $15 \mathrm{~g}$ Bacto agar) by successive cultures. Co-cultures were performed in triplicate on GA medium plates. On one side of the Petri dish, an agar plug with fresh fungal mycelium was inoculated and $10 \mu \mathrm{L}$ of Streptomyces spore suspension $\left(10^{5}\right.$ spores $\left./ \mathrm{mL}\right)$ were streaked on the opposite side (on approximately one third of the Petri dish, as illustrated in Figure 1A). The co-cultures were grown for 10 to 14 days at $25{ }^{\circ} \mathrm{C}$ in the dark. Controls were performed similarly but only with one microorganism.

\subsection{Metabolite Extraction}

Interaction zones (Figure 1A) from three independent Petri dishes were excised and pooled together in a flask with one volume of ethyl acetate $(99.8 \%$, Biosolve, Dieuze, France). After $90 \mathrm{~min}$ of agitation $(120 \mathrm{rpm})$ at room temperature, ethyl acetate extracts were recovered by filtering on a paper filter of $100 \mu \mathrm{m}$ and were vacuum-dried at room temperature. Dry extracts were resuspended at a concentration of $10 \mathrm{mg} / \mathrm{mL}$ either in DMSO (extra dry, 99.8\%, Biosolve) or in methanol (HPLC quality, 99.9\%, Carlo Erba, Val de Reuil, France). For fractioning experiments, 80 Petri dishes were pooled to obtain the final extract.

\subsection{Anti-Bacterial Screening}

Anti-bacterial screening tests were performed in 96-well plates by adding $5 \mu \mathrm{L}$ of extract to a $200 \mu \mathrm{L}$ culture $(0.05 \mathrm{OD} 600 \mathrm{~nm})$ of the Bacillus indicator strain. Culture growth was monitored every $10 \mathrm{~min}$ for $24 \mathrm{~h}\left(30^{\circ} \mathrm{C}\right.$, rapid shake mode) on a BioTech (Synergy HT, Winooski, U.S.A). The initial 64 different co-culture extracts were screened once against B. subtilis ATCC6633 and those with activity, as well as controls, were repeated at least five times independently. Antibacterial assays against other bacterial species (Staphylococcus aureus ATCC29213, Escherichia coli ATCC 25922, Enterococcus faecalis ATCC 292212, Pseudomonas aeruginosa ATCC 27853, Acinetobacter baumanii ATCC 19606, Klebsiella pneumonia 
ABC 42, and Enterococcus cloacae ABC 291) were performed on the ABC Platform (Université de Lorraine, Vandoeuvre-Lès-Nancy, France) according to the ISO 20776-1 protocol.

\subsection{GC-MS and LC-MS/MS Metabolite Profiling}

Dried extracts were silylated by adding $50 \mu \mathrm{L}$ of BSTFA/TMSCl (99/1) and heated at $50{ }^{\circ} \mathrm{C}$ for $12 \mathrm{~h}$. After evaporation of the derivatizing reagent, and the extract derivatives were solubilized in ethyl acetate and transferred for gas chromatography analysis. GC-MS analysis was performed on a Clarus 680 gas chromatograph coupled to a Clarus SQ8 quadrupole mass spectrometer (Perkin Elmer Inc., Waltham, MA, USA). Gas chromatography was carried out on a $\%$ diphenyl $/ 95 \%$ dimethyl polysiloxane fused-silica capillary column (DB-5 ms, $30 \mathrm{~m} \times 0.25 \mathrm{~mm}, 0.25 \mu \mathrm{m}$ film thickness, J\&W Scientific, Folsom, CA, USA) with helium as a carrier gas at a constant flow of $1 \mathrm{~mL} / \mathrm{min}$. The gas chromatograph was equipped with an electronically controlled split/splitless injection port. The injection $(1 \mu \mathrm{L})$ was performed at $250^{\circ} \mathrm{C}$ in the splitless mode. The oven temperature program was as follows: $80^{\circ} \mathrm{C}$ for $2 \mathrm{~min}$, increase from 80 to $190^{\circ} \mathrm{C}$ at a rate of $10^{\circ} \mathrm{C} / \mathrm{min}$, increase from 190 to $280^{\circ} \mathrm{C}$ at a rate of $15^{\circ} \mathrm{C} / \mathrm{min}$ and hold for $5 \mathrm{~min}$, then $10^{\circ} \mathrm{C} / \mathrm{min}$ until $300^{\circ} \mathrm{C}$ hold for $14 \mathrm{~min}$. Ionization was achieved under the electron impact mode $(70 \mathrm{eV}$ ionization energy). The source and transfer line temperatures were 180 and $250{ }^{\circ} \mathrm{C}$, respectively. Detection was carried out in scan mode: $\mathrm{m} / \mathrm{z} 45$ to $\mathrm{m} / \mathrm{z} 700$. The detector was switched off in the initial $2 \mathrm{~min}$ (solvent delay).

LC-MS/MS was performed with a Thermo Scientific ultimate 3000 liquid chromatography with a mass detector IonTrap LTQ Velos pro (Thermo Scientific, San Jose, CA, USA) fitted with an ESI source (capillary and source temperatures are 300 and $250{ }^{\circ} \mathrm{C}$, respectively). Detection was carried out in positive $(+5 \mathrm{kV})$ and in negative $(-4 \mathrm{kV})$ ion mode from $\mathrm{m} / \mathrm{z} 100$ to $\mathrm{m} / \mathrm{z} 2000$. The five most intense peaks in the MS spectra were submitted to fragmentation by CID at $45 \mathrm{eV}$-collision energy.

Separation was carried out with a C18 HypersiI gold aQ column $(100 \times 2.1 \mathrm{~mm}$ ID, $5 \mu$ particle, Thermo Fischer Scientific, Waltham, MA, USA). Analysis was performed in gradient mode at a flow rate of $200 \mu \mathrm{L} / \mathrm{min}$. The column was equilibrated during $20 \mathrm{~min}$ with $10 \%$ methanol, $5 \%$ acetonitrile and $85 \%$ water. After the sample injection $(5 \mu \mathrm{L})$, the gradient started from $10 \%$ to $50 \%$ of methanol, and from $5 \%$ to $50 \%$ of acetonitrile over $30 \mathrm{~min}$.

\subsection{Extract Fractioning}

Fractioning was performed with an HPLC Shimadzu LC-20AT coupled with a DAD SPD-20A detector. The semi-preparative column was in a Kinetex biphenyl stationary phase (300 mm* $4.6 \mathrm{~mm}, 5 \mu$ particle of 100 A, Phenomenex, Torrance, CA, USA). Analysis was performed in gradient mode at a flow rate of $1 \mathrm{~mL} / \mathrm{min}$. The gradient started from $0 \%$ to $100 \%$ methanol during $55 \mathrm{~min}$. The column was washed for $25 \mathrm{~min}$ with $100 \%$ water. All fractioning were realized four times with $100 \mu \mathrm{L}$ of $10 \mathrm{mg} / \mathrm{mL}$ extracts.

\subsection{Molecular Networking}

A molecular network was created using the online workflow (https://ccms-ucsd. github.io/GNPSDocumentation/) on the GNPS website (http://gnps.ucsd.edu). The data were filtered by removing all MS/MS fragment ions within $+/-17 \mathrm{Da}$ of the precursor $\mathrm{m} / \mathrm{z}$. MS/MS spectra were window-filtered by choosing only the top six fragment ions in the $+/-50$ Da window throughout the spectrum. The precursor ion mass tolerance was set to 2.0 Da and a MS/MS fragment ion tolerance of $0.5 \mathrm{Da}$. A network was created where edges were filtered to have a cosine score above 0.7 and more than six matched peaks. Furthermore, edges between two nodes were kept in the network if and only if each of the nodes appeared in each other's respective top 10 most similar nodes. Finally, the maximum size of a molecular family was set to 100 , and the lowest scoring edges were removed from molecular families until the molecular family size was below this threshold. The spectra in the network were then searched against GNPS' spectral libraries. The library 
spectra were filtered in the same manner as the input data. All matches kept between network spectra and library spectra were required to have a score above 0.7 and at least six matched peaks.

\subsection{Statistical Analysis}

PLS-DA and the heatmap were performed using the Metaboanalyst platform. Statistical analyses were $t$-tests and were performed with R 3.6.1 software.

Author Contributions: M.N., C.B., P.L. and E.G. designed the experiments; M.N. performed the fermentation experiments and the extraction and splitting procedures; M.N., S.D., A.Z. and P.C. performed data acquisition; M.N. performed the biological assays; M.N., E.G., C.B. and P.L. drafted the manuscript, and all authors revised and approved the final submitted manuscript. All authors have read and agreed to the published version of the manuscript.

Funding: This project was funded by the "Impact Biomolecules" project of the "Lorraine Université $\mathrm{d}^{\prime}$ Excellence" (in the context of the "Investissements d'avenir " program implemented by the French National Research Agency (ANR)).

Institutional Review Board Statement: Not applicable.

Informed Consent Statement: Not applicable.

Data Availability Statement: The data presented in this study are available on request from the corresponding author.

Acknowledgments: The authors acknowledge financial support from the "Impact Biomolecules" project of the "Lorraine Université d'Excellence" (in the context of the "Investissements d'avenir " program implemented by the French National Research Agency (ANR)).

Conflicts of Interest: The authors declare no conflict of interest.

\section{References}

1. Deveau, A.; Bonito, G.; Uehling, J.; Paoletti, M.; Becker, M.; Bindschedler, S.; Hacquard, S.; Hervé, V.; Labbé, J.; Lastovetsky, O.A.; et al. Bacterial-fungal interactions: Ecology, mechanisms and challenges. FEMS Microbiol. Rev. 2018, 42, 335-352. [CrossRef]

2. Velez, P.; Espinosa-Asuar, L.; Figueroa, M.; Gasca-Pineda, J.; Aguirre-von-Wobeser, E.; Eguiarte, L.E.; Hernandez-Monroy, A.; Souza, V. Nutrient dependent cross-kingdom interactions: Fungi and bacteria from an oligotrophic desert oasis. Front. Microbiol. 2018, 9. [CrossRef]

3. Frey-Klett, P.; Burlinson, P.; Deveau, A.; Barret, M.; Tarkka, M.; Sarniguet, A. Bacterial-fungal interactions: Hyphens between agricultural, clinical, environmental, and food microbiologists. Microbiol. Mol. Biol. Rev. 2011, 75, 583-609. [CrossRef]

4. Netzker, T.; Fischer, J.; Weber, J.; Mattern, D.J.; König, C.C.; Valiante, V.; Schroeckh, V.; Brakhage, A.A. Microbial communication leading to the activation of silent fungal secondary metabolite gene clusters. Front. Microbiol. 2015, 6. [CrossRef] [PubMed]

5. Martín, J.F.; Liras, P. Harnessing microbiota interactions to produce bioactive metabolites: Communication signals and receptor proteins. Curr. Opin. Pharmacol. 2019, 48, 8-16. [CrossRef] [PubMed]

6. Bérdy, J. Bioactive microbial metabolites: A personal view. J. Antibiot. 2005, 58, 1-26. [CrossRef] [PubMed]

7. Baltz, R.H. Gifted microbes for genome mining and natural product discovery. J. Ind. Microbiol. Biotechnol. 2017, 44, 573-588. [CrossRef] [PubMed]

8. Nicault, M.; Tidjani, A.-R.; Gauthier, A.; Dumarcay, S.; Gelhaye, E.; Bontemps, C.; Leblond, P. Mining the biosynthetic potential for specialized metabolism of a Streptomyces soil community. Antibiotics 2020, 9, 271. [CrossRef]

9. Waksman, S.A.; Harris, D.A.; Kupferberg, A.B.; Singher, H.O.; Styles, H. Streptocin, antibiotic isolated from mycelium of Streptomyces griseus, active against Trichomonas vaginalis, and certain bacteria. Exp. Biol. Med. 1949, 70, 308-312. [CrossRef]

10. Ikeda, H.; Nonomiya, T.; Usami, M.; Ohta, T.; Omura, S. Organization of the biosynthetic gene cluster for the polyketide anthelmintic macrolide avermectin in Streptomyces avermitilis. Proc. Natl. Acad. Sci. USA 1999, 96, 9509-9514. [CrossRef]

11. Ohnishi, Y.; Ishikawa, J.; Hara, H.; Suzuki, H.; Ikenoya, M.; Ikeda, H.; Yamashita, A.; Hattori, M.; Horinouchi, S. Genome sequence of the streptomycin-producing microorganism Streptomyces griseus IFO 13350. J. Bacteriol. 2008, 190, 4050-4060. [CrossRef] [PubMed]

12. Ōmura, S.; Ikeda, H.; Ishikawa, J.; Hanamoto, A.; Takahashi, C.; Shinose, M.; Takahashi, Y.; Horikawa, H.; Nakazawa, H.; Osonoe, T.; et al. Genome sequence of an industrial microorganism Streptomyces avermitilis: Deducing the ability of producing secondary metabolites. Proc. Natl. Acad. Sci. USA 2001, 98, 12215-12220. [CrossRef] [PubMed]

13. Bentley, S.D.; Chater, K.F.; Cerdeño-Tárraga, A.-M.; Challis, G.L.; Thomson, N.R.; James, K.D.; Harris, D.E.; Quail, M.A.; Kieser, H.; Harper, D.; et al. Complete genome sequence of the model actinomycete Streptomyces coelicolor A3 (2). Nature 2002, 417, 141-147. [CrossRef] [PubMed] 
14. Borodina, I. Genome-scale analysis of Streptomyces coelicolor A3 (2) metabolism. Genome Res. 2005, 15, 820-829. [CrossRef] [PubMed]

15. Rutledge, P.J.; Challis, G.L. Discovery of microbial natural products by activation of silent biosynthetic gene clusters. Nat. Rev. Microbiol. 2015, 13, 509-523. [CrossRef]

16. Brakhage, A.A.; Schroeckh, V. Fungal secondary metabolites-Strategies to activate silent gene clusters. Fungal Genet. Biol. 2011, 48, 15-22. [CrossRef]

17. Mao, D.; Okada, B.K.; Wu, Y.; Xu, F.; Seyedsayamdost, M.R. Recent advances in activating silent biosynthetic gene clusters in bacteria. Curr. Opin. Microbiol. 2018, 45, 156-163. [CrossRef]

18. Xia, H.; Li, X.; Li, Z.; Zhan, X.; Mao, X.; Li, Y. The application of regulatory cascades in Streptomyces: Yield enhancement and metabolite mining. Front. Microbiol. 2020, 11. [CrossRef]

19. Laureti, L.; Song, L.; Huang, S.; Corre, C.; Leblond, P.; Challis, G.L.; Aigle, B. Identification of a bioactive 51-membered macrolide complex by activation of a silent polyketide synthase in Streptomyces ambofaciens. Proc. Natl. Acad. Sci. USA 2011, 108, 6258-6263. [CrossRef]

20. Myronovskyi, M.; Luzhetskyy, A. heterologous production of small molecules in the optimized Streptomyces hosts. Nat. Prod. Rep. 2019, 36, 1281-1294. [CrossRef]

21. Ochi, K. Insights into microbial cryptic gene activation and strain improvement: Principle, application and technical aspects. J. Antibiot. 2017, 70, 25-40. [CrossRef] [PubMed]

22. Almeida, R.S.; Freitas, P.R.; Araújo, A.C.J.; Alencar Menezes, I.R.; Santos, E.L.; Tintino, S.R.; Moura, T.F.; Filho, J.R.; Ferreira, V.A.; Silva, A.C.A.; et al. GC-MS profile and enhancement of antibiotic activity by the essential oil of Ocotea odorifera and safrole: Inhibition of Staphylococcus aureus efflux pumps. Antibiotics 2020, 9, 247. [CrossRef] [PubMed]

23. Bertrand, S.; Bohni, N.; Schnee, S.; Schumpp, O.; Gindro, K.; Wolfender, J.-L. Metabolite induction via microorganism co-culture: A potential way to enhance chemical diversity for drug discovery. Biotechnol. Adv. 2014, 32, 1180-1204. [CrossRef] [PubMed]

24. Netzker, T.; Flak, M.; Krespach, M.K.; Stroe, M.C.; Weber, J.; Schroeckh, V.; Brakhage, A.A. Microbial interactions trigger the production of antibiotics. Curr. Opin. Microbiol. 2018, 45, 117-123. [CrossRef] [PubMed]

25. El Ariebi, N.; Hiscox, J.; Scriven, S.A.; Müller, C.T.; Boddy, L. Production and effects of volatile organic compounds during interspecific interactions. Fungal Ecol. 2016, 20, 144-154. [CrossRef]

26. Hiscox, J.; O'Leary, J.; Boddy, L. Fungus wars: Basidiomycete battles in wood decay. Stud. Mycol. 2018, 89, 117-124. [CrossRef] [PubMed]

27. Christofides, S.R.; Hiscox, J.; Savoury, M.; Boddy, L.; Weightman, A.J. Fungal control of early-stage bacterial community development in decomposing wood. Fungal Ecol. 2019, 42, 100868. [CrossRef]

28. Hervé, V.; Le Roux, X.; Uroz, S.; Gelhaye, E.; Frey-Klett, P. Diversity and structure of bacterial communities associated with Phanerochaete chrysosporium during wood decay. Environ. Microbiol. 2014, 16, 2238-2252. [CrossRef]

29. Johnston, S.R.; Boddy, L.; Weightman, A.J. Bacteria in decomposing wood and their interactions with wood-decay fungi. FEMS Microbiol. Ecol. 2016, 92, fiw179. [CrossRef]

30. Traxler, M.F.; Watrous, J.D.; Alexandrov, T.; Dorrestein, P.C.; Kolter, R. Interspecies interactions stimulate diversification of the Streptomyces coelicolor secreted metabolome. mBio 2013, 4. [CrossRef]

31. Ueda, K.; Kawai, S.; Ogawa, H.-O.; Kiyama, A.; Kubota, T.; Kawanobe, H.; Beppu, T. Wide distribution of interspecific stimulatory events on antibiotic production and sporulation among Streptomyces species. J. Antibiot. 2000, 53, 979-982. [CrossRef] [PubMed]

32. Charusanti, P.; Fong, N.L.; Nagarajan, H.; Pereira, A.R.; Li, H.J.; Abate, E.A.; Su, Y.; Gerwick, W.H.; Palsson, B.O. Exploiting adaptive laboratory evolution of Streptomyces clavuligerus for antibiotic discovery and overproduction. PLoS ONE 2012, 7, e33727. [CrossRef] [PubMed]

33. Luti, K.J.K.; Mavituna, F. Elicitation of Streptomyces coelicolor with dead cells of bacillus subtilis and Staphylococcus aureus in a bioreactor increases production of undecylprodigiosin. Appl. Microbiol. Biotechnol. 2011, 90, 461-466. [CrossRef] [PubMed]

34. Onaka, H.; Mori, Y.; Igarashi, Y.; Furumai, T. Mycolic acid-containing bacteria induce natural-product biosynthesis in Streptomyces species. Appl. Environ. Microbiol. 2011, 77, 400-406. [CrossRef] [PubMed]

35. Pérez, J.; Muñoz-Dorado, J.; Braña, A.F.; Shimkets, L.J.; Sevillano, L.; Santamaría, R.I. Myxococcus xanthus induces actinorhodin overproduction and aerial mycelium formation by Streptomyces coelicolor. Microbiol. Biotechnol. 2011, 4, 175-183. [CrossRef]

36. Wakefield, J.; Hassan, H.M.; Jaspars, M.; Ebel, R.; Rateb, M.E. Dual induction of new microbial secondary metabolites by fungal bacterial co-cultivation. Front. Microbiol. 2017, 8. [CrossRef] [PubMed]

37. Schroeckh, V.; Scherlach, K.; Nutzmann, H.-W.; Shelest, E.; Schmidt-Heck, W.; Schuemann, J.; Martin, K.; Hertweck, C.; Brakhage, A.A. Intimate bacterial-fungal interaction triggers biosynthesis of archetypal polyketides in Aspergillus nidulans. Proc. Natl. Acad. Sci. USA 2009, 106, 14558-14563. [CrossRef]

38. Hervé, V.; Ketter, E.; Pierrat, J.-C.; Gelhaye, E.; Frey-Klett, P. Impact of Phanerochaete chrysosporium on the functional diversity of bacterial communities associated with decaying wood. PLoS ONE 2016, 11. [CrossRef]

39. Johnston, S.R.; Hiscox, J.; Savoury, M.; Boddy, L.; Weightman, A.J. Highly competitive fungi manipulate bacterial communities in decomposing beech wood (Fagus sylvatica). FEMS Microbiol. Ecol. 2019, 95. [CrossRef]

40. Aqueveque, P.; Céspedes, C.L.; Becerra, J.; Aranda, M.; Sterner, O. Antifungal activities of secondary metabolites isolated from liquid fermentations of Stereum hirsutum (Sh134-11) against Botrytis cinerea (grey mould agent). Food Chem. Toxicol. 2017, 109, 1048-1054. [CrossRef] 
41. Cilerdzic, J.; Stajic, M.; Vukojevic, J. Potential of submergedly cultivated mycelia of Ganoderma spp. as antioxidant and antimicrobial agents. Curr. Pharm. Biotechnol. 2016, 17, 275-282. [CrossRef]

42. Han, J.-J.; Bao, L.; He, L.-W.; Zhang, X.-Q.; Yang, X.-L.; Li, S.-J.; Yao, Y.-J.; Liu, H. Phaeolschidins A-E, five hispidin derivatives with antioxidant activity from the fruiting body of Phaeolus schweinitzii collected in the Tibetan Plateau. J. Nat. Prod. 2013, 76, 1448-1453. [CrossRef] [PubMed]

43. Knežević, A.; Stajić, M.; Sofrenić, I.; Stanojković, T.; Milovanović, I.; Tešević, V.; Vukojević, J. Antioxidative, antifungal, cytotoxic and antineurodegenerative activity of selected trametes species from Serbia. PLoS ONE 2018, 13, e0203064. [CrossRef] [PubMed]

44. Riley, R.; Salamov, A.A.; Brown, D.W.; Nagy, L.G.; Floudas, D.; Held, B.W.; Levasseur, A.; Lombard, V.; Morin, E.; Otillar, R.; et al. Extensive sampling of basidiomycete genomes demonstrates inadequacy of the white-rot/brown-rot paradigm for wood decay fungi. Proc. Natl. Acad. Sci. USA 2014, 111, 9923-9928. [CrossRef]

45. Moody, S.C. Microbial co-culture: Harnessing intermicrobial signaling for the production of novel antimicrobials. Future Microbiol. 2014, 9, 575-578. [CrossRef] [PubMed]

46. Tian, M.; Zhao, P.; Li, G.; Zhang, K. In depth natural product discovery from the basidiomycetes Stereum species. Microorganisms 2020, 8, 1049. [CrossRef]

47. Oppong-Danquah, E.; Budnicka, P.; Blümel, M.; Tasdemir, D. Design of fungal co-cultivation based on comparative metabolomics and bioactivity for discovery of marine fungal agrochemicals. Mar. Drugs 2020, 18, 73. [CrossRef]

48. Fischer, J.; Müller, S.Y.; Netzker, T.; Jäger, N.; Gacek-Matthews, A.; Scherlach, K.; Stroe, M.C.; García-Altares, M.; Pezzini, F.; Schoeler, H.; et al. Chromatin mapping identifies BasR, a key regulator of bacteria-triggered production of fungal secondary metabolites. eLife 2018, 7, e40969. [CrossRef]

49. Khalil, Z.G.; Cruz-Morales, P.; Licona-Cassani, C.; Marcellin, E.; Capon, R.J. Inter-kingdom beach warfare: Microbial chemical communication activates natural chemical defences. ISME J. 2019, 13, 147-158. [CrossRef]

50. Krause, K.; Jung, E.-M.; Lindner, J.; Hardiman, I.; Poetschner, J.; Madhavan, S.; Matthäus, C.; Kai, M.; Menezes, R.C.; Popp, J.; et al. Response of the wood-decay fungus Schizophyllum commune to co-occurring microorganisms. PLoS ONE 2020, 15. [CrossRef]

51. Tillotson, R.D.; Wösten, H.A.B.; Richter, M.; Willey, J.M. A surface active protein involved in aerial hyphae formation in the filamentous fungus Schizophillum commune restores the capacity of a bald mutant of the filamentous bacterium Streptomyces coelicolor to erect aerial structures. Mol. Microbiol. 1998, 30, 595-602. [CrossRef] [PubMed]

52. Saravana Kumar, P.; Al-Dhabi, N.A.; Duraipandiyan, V.; Balachandran, C.; Praveen Kumar, P.; Ignacimuthu, S. In vitro antimicrobial, antioxidant and cytotoxic properties of Streptomyces lavendulae strain SCA5. BMC Microbiol. 2014, 14, 291. [CrossRef] [PubMed]

53. Halket, J.M.; Waterman, D.; Przyborowska, A.; Patel, R.K.P.; Fraser, P.D.; Bramley, P.M. Chemical derivatization and mass spectral libraries in metabolic profiling by GC/MS and LC/MS/MS. J. Exp. Bot. 2005. [CrossRef] [PubMed]

54. Gurudeeban, S.; Ramanathan, T.; Satyavani, K. Antimicrobial and radical scavenging effects of alkaloid extracts from Rhizophora mucronata. Pharm. Chem. J. 2013, 47, 50-53. [CrossRef] 